\title{
Control of the stop band of an acoustic double fishnet
}

\author{
Alasdair R. J. Murray, ${ }^{\text {a) }}$ Euan Hendry, Ian R. Summers, J. Roy Sambles, and \\ Alastair P. Hibbins \\ Electromagnetic and Acoustic Materials, Department of Physics and Astronomy, University of Exeter, \\ Stocker Road, Exeter, EX4 4QL, United Kingdom
}

(Received 15 April 2013; revised 21 June 2013; accepted 23 July 2013)

\begin{abstract}
The acoustic transmittance of two closely spaced solid plates, each perforated with a square array of cylindrical holes, exhibits a band of near-perfect acoustic attenuation originating from hybridization between a resonance in the gap separating the plates and pipe resonances in the holes. Displacement of one plate relative to the other, such that the holes are no longer aligned, or an increase in the plate separation leads to an increased center frequency of the stop band. This ability to easily tune the frequency of the stop band may prove advantageous.
\end{abstract}

(C) 2013 Acoustical Society of America. [http://dx.doi.org/10.1121/1.4817898]

PACS number(s): 43.20.Mv, 43.20.El [ANN]

Pages: 1754-1759

\section{INTRODUCTION}

In air, solid bodies are generally effective at blocking the transmission of sound. The addition of slits or holes provides a mechanism for the flow of air through the structure but also permits the potentially unwanted transmission of sound. Studies of the acoustic response of such perforated solid bodies have shown that at certain frequencies, dictated by the slit (or hole) depth there may be near unity transmission of sound. ${ }^{1-3}$ Another study ${ }^{4}$ has shown that perforated plates may suppress the transmission of sound better than an entirely solid plate of real (i.e., not perfectly rigid) material across a narrow frequency band owing to diffraction effects. More recently, the response of two non-diffracting solid perforated plates separated by a small gap has been explored. The resonant mode of the gap, defined by the pitch of the hole array, is the origin of a band of near-perfect acoustic attenuation. $^{5,6}$ An experimental study ${ }^{7}$ of this structure, referred to as the acoustic double fishnet (ADF), has confirmed the existence of the stop band; a transmission minimum of around $-40 \mathrm{~dB}$ was observed in the region of $19 \mathrm{kHz}$, as dictated by the pitch of the hole array. Varying the pitch of the hole array offers one method for controlling the stop band frequency. However, it is obviously difficult to vary the pitch of a hole array once it has been made. In the present study, the exploration of the ADF structure is extended, quantifying the effects of varying the in-plane displacement of one plate relative to the other or the separation between the plates.

The experimental sample (Fig. 1) consists of two perspex (Lucite) plates, each of thickness $h_{\mathrm{m}}=12 \mathrm{~mm}$, perforated with circular holes of radius $r=1.2 \mathrm{~mm}$ in a square array of pitch $\Lambda=8 \mathrm{~mm}$. The plates are separated by a gap $h_{\mathrm{g}}$ which is set at $0.94 \mathrm{~mm}$ for the displacement experiment. The sample size is $200 \mathrm{~mm} \times 200 \mathrm{~mm}$ and is placed in an equivalent sized aperture within a solid material. An aluminum mirror collimates sound from a loudspeaker, small

\footnotetext{
a) Author to whom correspondence should be addressed. Electronic mail: a.r.j.murray@exeter.ac.uk
}

enough to be approximated as a point source, placed at its focus. The collimated planar beam of sound is incident normal to the sample. A second mirror focuses the transmitted sound onto an electret microphone. The investigation is undertaken in air where, for the frequency regime considered, the perspex plates are assumed to approximate to perfectly rigid bodies. The transmittance is determined by normalizing the detected data to the transmitted data when no sample is present in the aperture.

\section{DIAGONAL AND LATERAL PLATE MISALIGNMENT}

With the holes aligned, i.e., the in-plane displacement $h_{\text {off }}$ is zero; the transmission of sound at normal incidence shows a series of peaks, associated with pipe resonances from the holes running through the total thickness of the ADF structure. Odd-order modes, possessing a pressure antinode at the mid-point of the structure are shifted in frequency due to volume flow leakage into the gap region, whereas even-order modes have a pressure antinode at the gap and are not significantly affected by the presence of the gap. $^{7}$

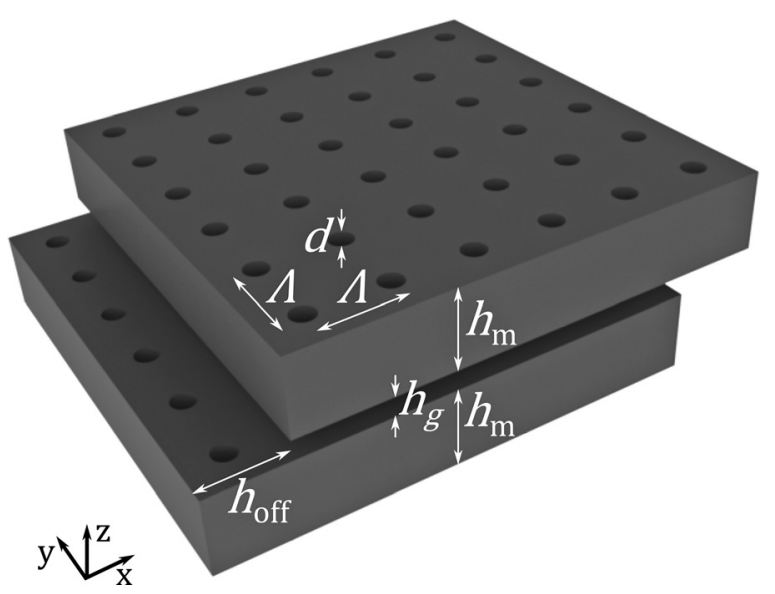

FIG. 1. Schematic of the ADF structure where $h_{\mathrm{m}}$ is the plate thickness and $h_{\mathrm{g}}$ is the gap between the plates. Cylindrical holes with diameter $d$ are in a square array of pitch $\Lambda$. The displacement between the plates is $h_{\text {off }}$ (lateral displacement shown; diagonal displacement also investigated). 

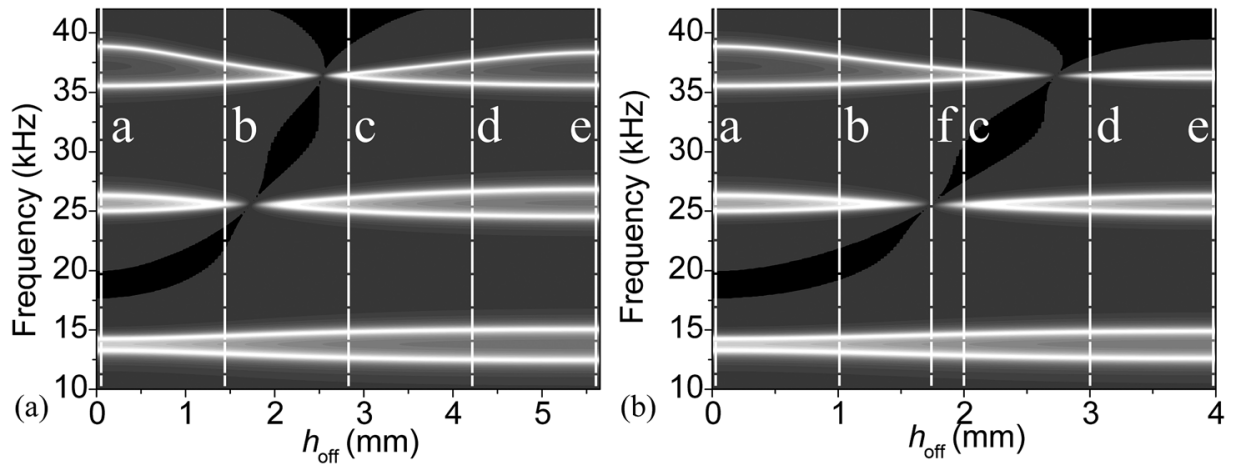

FIG. 2. Modeled transmission spectra for two perspex plates (thickness $h_{\mathrm{m}}$ $=12 \mathrm{~mm}, d=2.4 \mathrm{~mm}$, and $\Lambda=8 \mathrm{~mm}$ ) separated by $h_{\mathrm{g}}=0.94 \mathrm{~mm}$, for the cases of (a) a diagonal offset, and (b) a lateral offset. White regions indicate high transmission and black regions indicate $<0.5 \%$ amplitude transmission. Dashed lines indicate the diagonal and lateral offsets for which experimental data were taken.
The effect on the acoustic transmittance of misaligning the plates by a diagonal displacement $h_{\text {off }}$ in the $x y$-plane is first considered, i.e., displacement $h_{\text {off }} / \sqrt{2}$ in both $x$ - and $y$-directions. The acoustic response of the structure can be predicted using a modified version of the modal matching technique described by Bell et ll $^{7} \mathrm{We}$ approximate the perspex plates to be perfectly rigid and as such no acoustic waves are supported within the plate. Figure 2(a) shows modeled transmission spectra as a function of diagonal displacement. [Equivalent data calculated using a finite element method (FEM) modeling package (COMSOL multiphysics) agrees with that calculated from the modal matching method.] It is apparent that, as the plates are progressively offset, the stop band is shifted to higher frequency. In addition, the changing geometry has some effect on the frequencies of the transmission resonances.

Figure 2(b) shows transmission spectra for the case when the plates are offset laterally by $h_{\text {off }}$ in the $x$ or $y$ direction.

It is apparent from Figs. 2(a) and 2(b) that the profile of the stop band is almost identical in the diagonal and lateral offset cases (e.g., compare the offsets and frequencies at which the stop band crosses the transmission maxima, at approximately $1.7 \mathrm{~mm} / 25 \mathrm{kHz}$ and $2.6 \mathrm{~mm} / 38 \mathrm{kHz}$ in both cases).
In the corresponding experimental studies, for diagonal offset the frequency is varied from $10 \mathrm{kHz}$ to $40 \mathrm{kHz}$ and the plates offset by integer multiples of $1.42 \mathrm{~mm}$ (i.e., $1 \mathrm{~mm}$ in both $x$ and $y$ ); for lateral offset the same frequency range is used and the plates are offset by integer multiples of $1 \mathrm{~mm}$, with additional data recorded for an offset of $1.74 \mathrm{~mm}$ to demonstrate the suppression of the transmission resonances otherwise seen at $25 \mathrm{kHz}$. The offsets used in the experimental studies are indicated by the vertical dashed lines in the two panels of Fig. 2. The experimental transmittance is compared with the predicted transmittance from the modal matching code in Figs. 3(a)-3(e) for the case of diagonal offset and Figs. 4(a)-4(f) for the case of lateral offset. For both sets of data, the frequencies and shapes of the experimental transmission resonances agree well with those predicted by the modal matching method. The experimental transmission peaks are lower than predicted-this discrepancy may be attributed to viscous losses that are not included within the modeling. The transmittance in the region of the stop band is around $-40 \mathrm{~dB}$; the predicted complete blockage of sound is not achieved; this again may be attributed to viscous losses. Figure 4(f) specifically shows the transmission for a lateral offset of $1.74 \mathrm{~mm}$ where the stop band coincides with the transmission resonance observed at around $25 \mathrm{kHz}$.
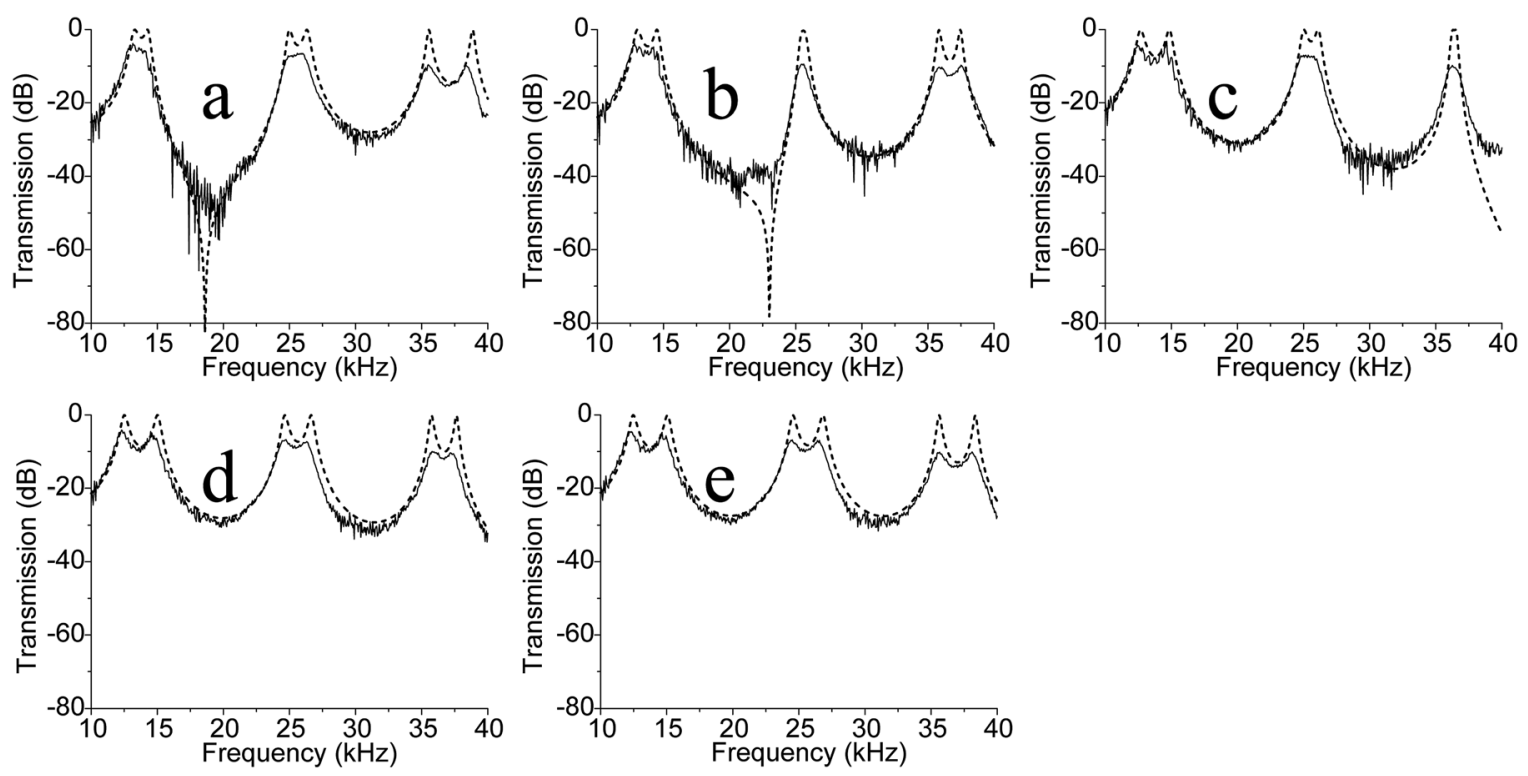

FIG. 3. Experimental (solid line) and modeled (dashed line) transmission results through the ADF structure in air. Shown are the transmission for a diagonal offset of $h_{\text {off }}=$ (a) $0 \mathrm{~mm}$, (b) $1.4 \mathrm{~mm}$, (c) $2.8 \mathrm{~mm}$, (d) $4.2 \mathrm{~mm}$, and (e) $5.6 \mathrm{~mm}$, respectively. 

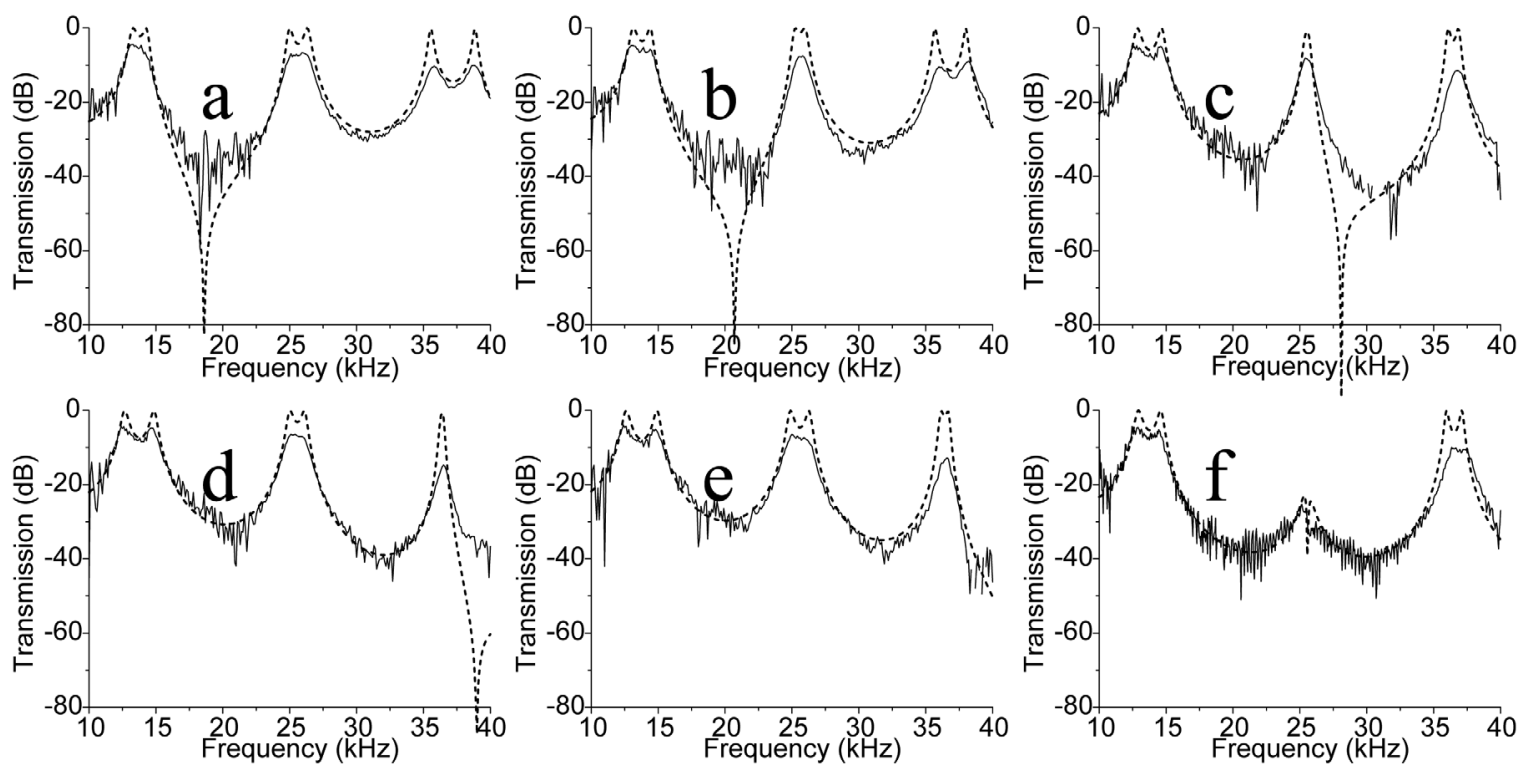

FIG. 4. Experimental (solid line) and modeled (dashed line) transmission results through the ADF structure in air. Shown are the transmission for a lateral offset of $h_{\text {off }}=$ (a) $0.0 \mathrm{~mm}$, (b) $1.0 \mathrm{~mm}$, (c) $2.0 \mathrm{~mm}$, (d) $3.0 \mathrm{~mm}$, (e) $4.0 \mathrm{~mm}$, and (f) $1.74 \mathrm{~mm}$, respectively.

To further elucidate this behavior an approximate analytical model is now outlined, following a similar procedure to that used by Bell et al. ${ }^{7}$ for aligned plates. For the misaligned case, a square unit cell is chosen centered on a hole in the "input" side of the plate. The acoustic pressure in the gap region is approximated as a sum of zero-order Bessel functions, centered on the "input" hole, of the form

$$
p(r)=A J_{0}(k r)+B Y_{0}(k r),
$$

where $A$ and $B$ are constants, $k$ is the wave number, and $r$ is the distance from the center of the input hole. Symmetry conditions force an antinode at the (square) unit-cell boundary, hence for simplicity a square geometry is assumed for the acoustic-pressure wave fronts in the gap region. The transmission through the structure is determined by the amplitude of this pressure field at the position of the "output" hole, with the condition for zero transmission being that the acoustic pressure in the gap exhibits a nodal line that runs through the position of the output hole. As the offset of the plates is increased, the distance between the output hole and the unit-cell boundary is decreased; to achieve zero transmission a shorter distance between the nodal line and the boundary antinode is required, and hence a higher frequency. Figure 5 shows the predictions of this approximate analytical model for the cases of a diagonal offset and a lateral offset. In addition to the variation of the stop-band frequency with hole offset, the figure shows estimated changes in the transmission bands; mode splitting is assumed to be proportional to the strength of the coupling between the holes in one plate and the holes in the other (determined by the amplitude of the pressure field in the gap region at the position of the output hole) and hence falls to zero at the stop-band frequency. (To avoid over-complication of the analytical model, it is assumed that changes in the extent of mode splitting affect only the higher-frequency mode of a pair while the lowerfrequency mode of the pair remains at a fixed frequency.)
For the case of diagonal misalignment, the modalmatching results [Fig. 2(a)] and the results from the analytical model [Fig. 5(a)] are in good agreement, despite the approximations involved in the analytical calculation; [the stop band crosses the transmission maxima in Fig. 2(a) at offsets equivalent to $h_{\text {off }} / \Lambda=0.22$ and 0.32 ; the crossings in Fig. 5(a) are at $h_{\text {off }} / \Lambda=0.20$ and 0.31]. For the case of lateral misalignment, the modal-matching results [Fig. 2(b)] and the results from the analytical model [Fig. 5(b)] show similar behavior, but values of $h_{\text {off }} / \Lambda$ from the analytical model are smaller than corresponding values from modal matching by a factor of approximately 1.50; [the stop band crosses the transmission maxima in Fig. 2(b) at offsets equivalent to $h_{\text {off }} / \Lambda=0.22$ and 0.34; the crossings in Fig. 5(b) are at $h_{\text {off }} /$ $\Lambda=0.14$ and 0.22$]$. This difference between the diagonal and lateral cases is because the analytical model, as mentioned above, uses a square geometry for the pressure field in the gap and hence assumes square nodal lines, whereas results from FEM modeling in Fig. 6 show the nodal lines to be approximately circular - in the case of lateral misalignment, this introduces an erroneous factor of $\sqrt{2}$ into the analytical modeling. The six panels in Fig. 6 show FEM plots of
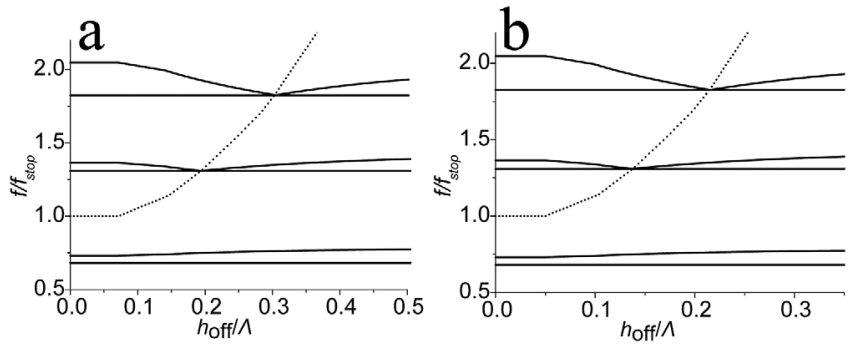

FIG. 5. Transmission maxima (solid lines) and transmission minimum (solid line with circular markers) calculated from the analytical model, for the cases of (a) a diagonal offset, and (b) a lateral offset. The $y$ axis, labeled " $\mathrm{f} / \mathrm{f}_{\text {stop }}$ " corresponds to the sound frequency divided by the frequency of the stop band. 

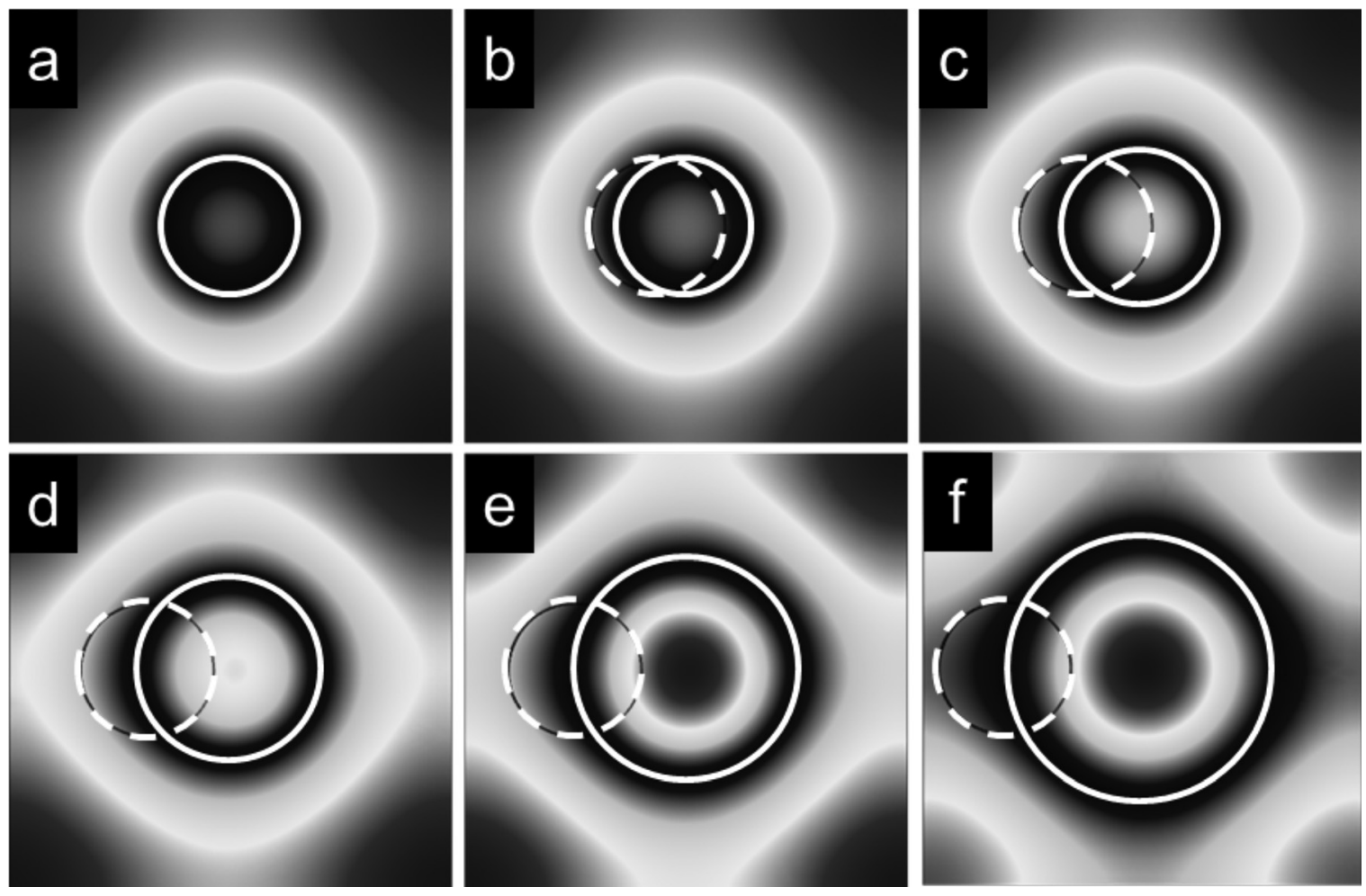

FIG. 6. FEM results for the time-averaged pressure field in the center of the gap between the two plates for $h_{\text {off }}=(\mathrm{a}) 0.0 \mathrm{~mm}$, (b) $0.5 \mathrm{~mm}$, (c) $1.0 \mathrm{~mm}$, (d) $1.5 \mathrm{~mm}$, (e) $2.0 \mathrm{~mm}$, (f) $2.5 \mathrm{~mm}$ at the respective frequencies of the stop band. White to black represents high to low amplitude. Each panel represents a square unit cell of side $8 \mathrm{~mm}$.

the time-averaged pressure field in the $x y$-plane through the center of the gap region for various values of $h_{\text {off }}$ (lateral displacement). The plots are taken at the respective stop-band frequencies for each value of $h_{\text {off }}$. In each plot it can be seen that a pressure node (marked by a solid white circle) runs through the position of the output hole (marked by a dashed white circle) confirming the previous suggestion that this is the condition for sound blockage.

\section{EFFECT OF VARYING THE GAP BETWEEN ALIGNED PLATES}

Figure 7(a) shows modal-matching results for transmission through two aligned plates ( $h_{\text {off }}$ set to zero) as a function of the plate separation $h_{\mathrm{g}}$. As $h_{\mathrm{g}}$ is increased the frequency of the stop band is shifted to higher frequencies. The pattern of transmission maxima and minima in Fig. 7(a) can be understood as follows: For smaller values of plate separation $h_{\mathrm{g}}$ the system corresponds to that analyzed by Bell et $\mathrm{al}^{7}$ and can be explained in terms of a two-dimensional model of the sound field in the gap in which the acoustic pressure is represented by an expression of the same form as that in Eq. (1). The frequency of the transmission minimum corresponds to the fundamental resonance in the gap and is determined by the pitch $\Lambda$ of the hole array; the frequencies of the transmission maxima are determined by the pipe resonances in the holes, which are affected by "leakage" of air flow into the gap. For larger values of plate separation $h_{\mathrm{g}}$, in each unit cell the hole-gap-hole system can be modeled as a pipe of varying cross-section whose resonance frequencies
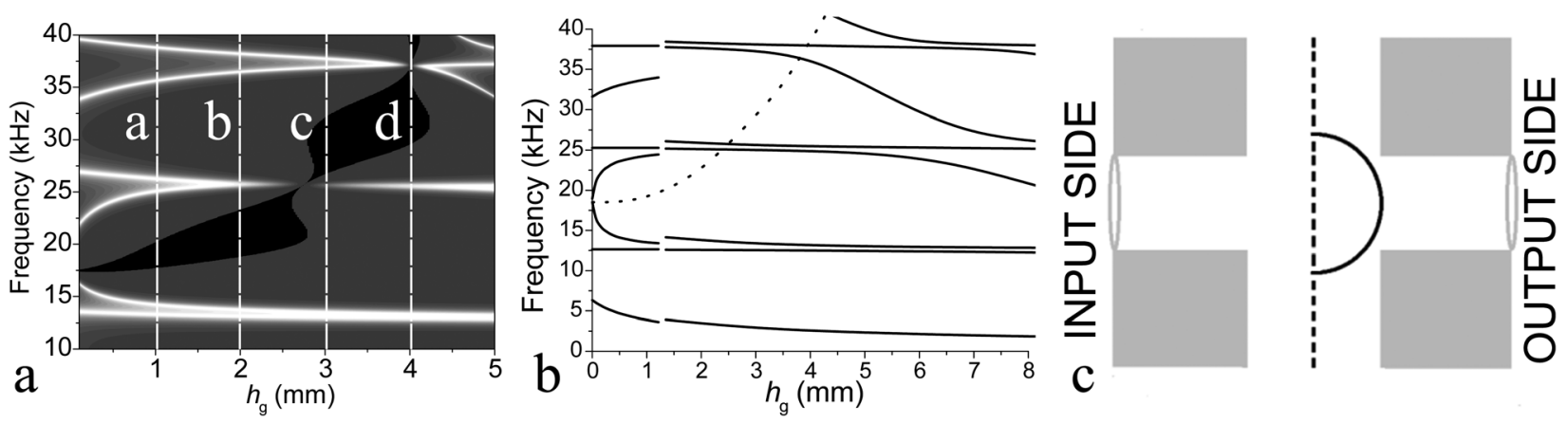

FIG. 7. (a) Modeled transmission from the modal matching method showing the transmission of two $12 \mathrm{~mm}$ thick, aligned plates as a function of the gap $h_{\mathrm{g}}$. White regions indicate high transmission and black regions indicate $<0.5 \%$ amplitude transmission. The dashed lines labeled (a)-(d) correspond to the experimentally measured gap sizes in Fig. 9. (b) Graph showing transmission maxima (solid lines) and minimum (line with circular markers) predicted by the three analytical models. (c) Schematic diagram showing the shape of the nodal surface assumed when calculating the frequency of the transmission minimum. 


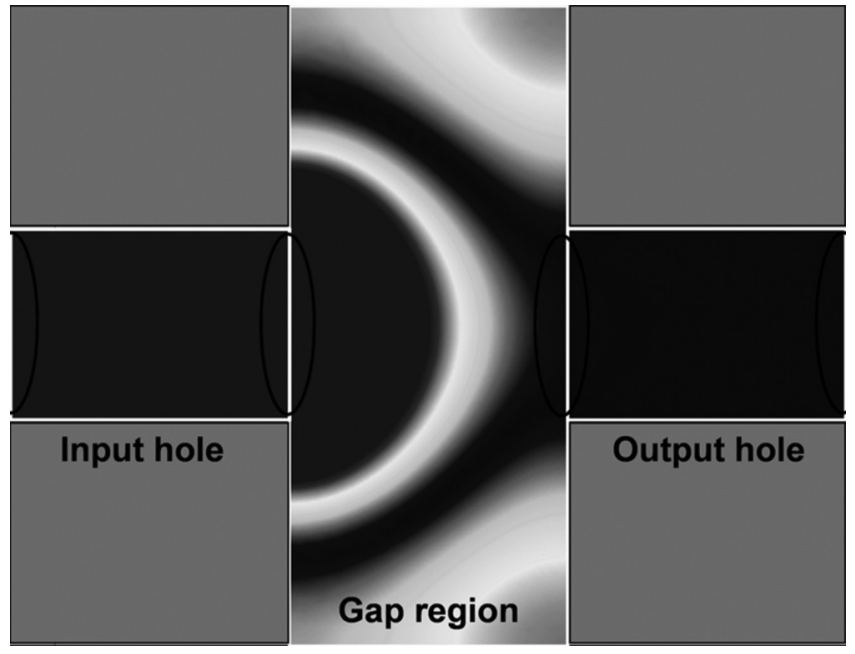

FIG. 8. FEM plot of the time-averaged pressure field inside the gap region for a gap of $h_{\mathrm{g}}=3.5 \mathrm{~mm}$, at the frequency of the transmission minimum $(33.1 \mathrm{kHz})$. The grayscale has been saturated to clarify the pressure node at the entrance of the output hole. White to black represents high to low amplitude.

(end-to-end modes) can be readily calculated. ${ }^{8}$ Figure $7(\mathrm{~b})$ shows the predictions of these two analytical models-for smaller and larger values of $h_{\mathrm{g}}$ (full lines). Missing from this analysis so far is a consideration of how the frequency of the transmission minimum varies with plate separation. This requires a three-dimensional model of the sound field in the gap. The line with circular markers in Fig. 7(b) shows predictions of the transmission minimum, calculated using a third analytical model, based on the simplifying assumption that the acoustic pressure on the central plane of the gap (i.e., mid-way between the plates) follows the two dimensional model of Bell et al., ${ }^{7}$ i.e., of the same form as that given in Eq. (1). It is further assumed that the principal nodal line in this plane extends into the "output" half of the gap as a nodal surface with a semicircular profile [Fig. 7(c)]. The condition for a transmission minimum is that the nodal surface lies at the entrance to the output hole, i.e., the radius of the semicircular profile is equal to half the plate separation $h_{\mathrm{g}}$. On the central plane of the gap, this condition fixes the position of the nodal line in relation to the pressure antinode at the unit-cell boundary - as the plate separation is increased, a shorter distance between the nodal line and the boundary antinode, and hence a higher frequency, is required to achieve a transmission minimum. [The reader will observe that this analysis is very close to the analysis presented above for the case of misaligned plates; this provides an explanation for the similarity between the variation of stopband frequency with plate offset (Figs. 2 and 5) and its variation with plate separation (Fig. 7).] Note that in Fig. 7(b) there is no attempt to combine the three models that produce the various lines, i.e., no attempt to model the interaction between the processes which produce the transmission maxima and those which produce the stop band.

The modal-matching results [Fig. 7(a)] and the results from the analytical models [Fig. 7(b)] show broadly similar behavior, despite the significant approximations involved in the analytical calculations. This suggests that the insights provided by the analytical models are valid for interpretation of the transmission maxima and minima in the modalmatching results. Figure 8 shows FEM results for the timeaveraged pressure field in the $x z$-plane for a plate separation $h_{\mathrm{g}}=3.5 \mathrm{~mm}$ at the frequency of sound blockage. A pressure node can be seen at the entrance to the output hole, but note that the nodal surface has a more complex shape than that assumed in the analytical model. The experimental sample and method are as described for the first experiment. The plate separation is varied from $0.94 \mathrm{~mm}$ to $3.76 \mathrm{~mm}$ by increasing the number of spacers between the plates in $0.94 \mathrm{~mm}$ steps. The separations used in the experimental
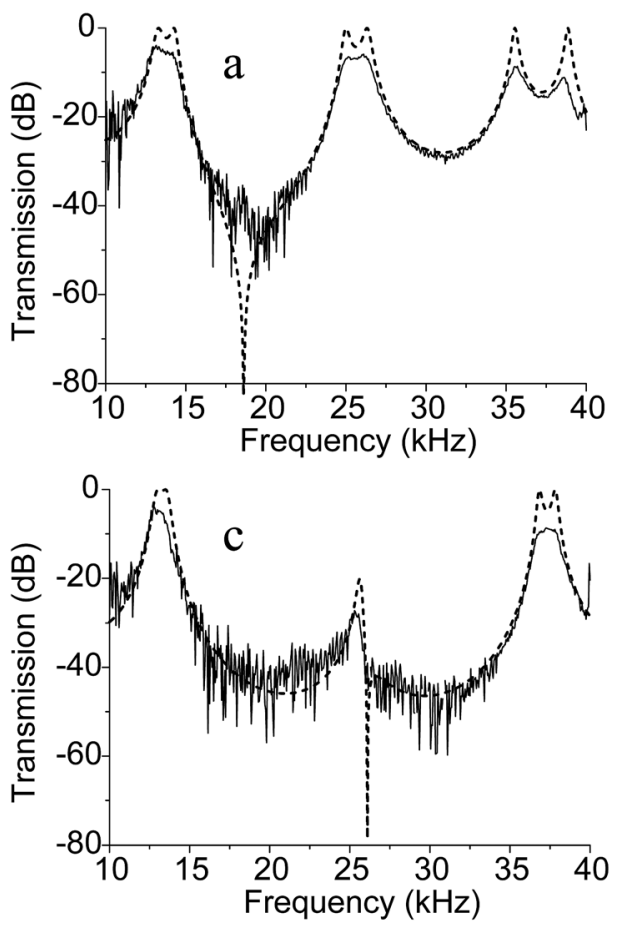
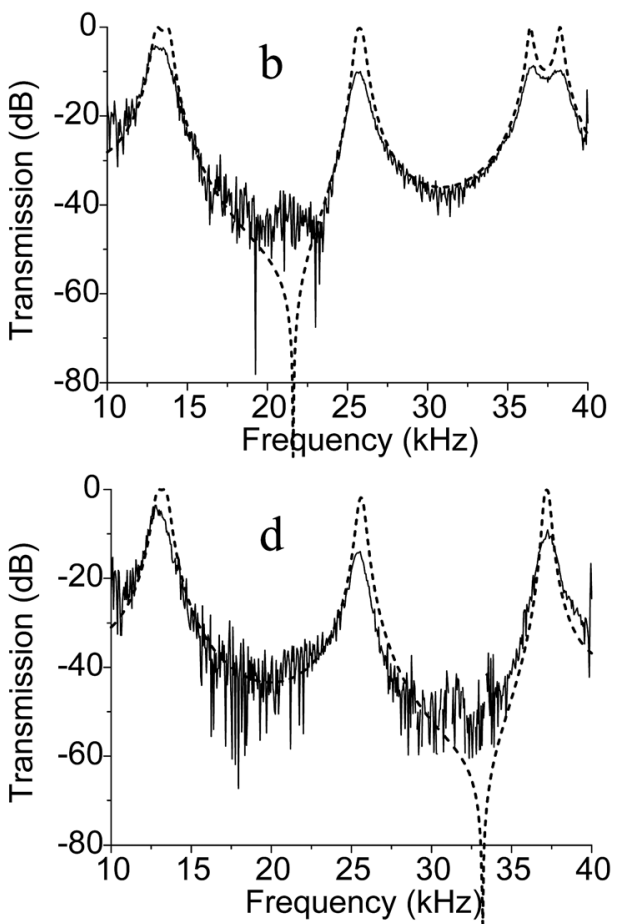

FIG. 9. Experimental (solid) and modeled (dashed line) transmission results through the ADF structure in air as the plate separation is varied. Shown are the transmission for $h_{\mathrm{g}}=$ (a) $0.94 \mathrm{~mm}$, (b) $1.88 \mathrm{~mm}$, (c) $2.82 \mathrm{~mm}$, (d) $3.76 \mathrm{~mm}$, respectively. 
studies are indicated by the vertical dashed lines in Fig. 7(a). In Fig. 9, the experimental transmittance is compared with the transmittance determined from the modal-matching code, with generally good agreement; (FEM results, not shown, are similar to the modal-matching predictions). For the smaller gap sizes a band of strongly suppressed transmission is clearly demonstrated towards the lower end of the measured frequency range and, as the gap size is increased, this stop band moves to higher frequencies. As in the first experiment, the predicted complete blockage of sound is not achieved experimentally, presumably because of viscous losses. In Fig. 9(c), for $h_{\mathrm{g}}=3 \mathrm{~mm}$ the center of the stop band lies very close in frequency to the transmission resonance at $25 \mathrm{kHz}$ [see Fig. 7(a)] and the latter is strongly suppressed.

\section{CONCLUSION}

The acoustic double fishnet structure allows for the flow of air from one side to the other while simultaneously offering a band of sound suppression at a frequency determined by the geometry. This stopband can be easily tuned by either misaligning the plates or by varying the gap between them. The stopband frequency is shifted to higher frequencies by in-plane displacement of one plate or by increasing the plate separation. When the stopband has been shifted such that it falls at the same frequency as a transmission maximum, that maximum is suppressed. For the $12 \mathrm{~mm}$ thickness plates tested, the modes at $25 \mathrm{kHz}$ are almost entirely suppressed at an in-plane displacement of $1.74 \mathrm{~mm}$. Similarly the same mode suppression can be achieved with aligned plates at a separation of $h_{\mathrm{g}}=2.8 \mathrm{~mm}$. Using either in-plane displacement or increased plate separation to suppress the $25 \mathrm{kHz}$ resonances [data in Fig. 4(f) and Fig. 9(c)] results in transmission of less than $-30 \mathrm{~dB}$ over the remarkably broad range of 17 to $35 \mathrm{kHz}$. Bell et al. ${ }^{7}$ have suggested that transmission resonances can be suppressed by choosing a suitable plate thickness; however, results from the present paper show how this can be achieved by simply changing the relative position of the plates. Varying the plate separation may be a more attractive option than in-plane displacement of one plate in cases where it is desirable to maintain an unimpeded airflow through the structure.

\section{ACKNOWLEDGMENTS}

The authors acknowledge funding of ARJM by EPSRC and Sonardyne International. The authors would like to thank the workshop technicians N. Cole, K. White, and M. Wears for expert manufacture of the experimental apparatus.

${ }^{1}$ M.-H. Lu, X.-K. Liu, L. Feng, J. Li, C.-P. Huang, Y.-F. Chen, Y.-Y. Zhu, S.-N. Zhu, and N.-B. Ming, "Extraordinary acoustic transmission through a 1d grating with very narrow apertures," Phys. Rev. Lett. 99, 174301 (2007).

${ }^{2}$ L. Zhou and G. A. Kriegsmann, "Complete transmission through a periodically perforated rigid slab," J. Acoust. Soc. Am. 121, 3288-3299 (2007).

${ }^{3}$ J. Christensen, L. Martin-Moreno, and F. J. Garcia-Vidal, "Theory of resonant acoustic transmission through subwavelength apertures," Phys. Rev. Lett. 101, 014301 (2008).

${ }^{4}$ H. Estrada, P. Candelas, A. Uris, F. Belmar, F. J. García de Abajo, and F. Meseguer, "Extraordinary sound screening in perforated plates," Phys. Rev. Lett. 101, 084302 (2008).

${ }^{5}$ Z. Liu and G. Jin, "Acoustic transmission resonance and suppression through double-layer subwavelength hole arrays," J. Phys. 22, 305003 (2010).

${ }^{6}$ J. Christensen, L. Martin-Moreno, and F. J. Garcia-Vidal, “All-angle blockage of sound by an acoustic double-fishnet metamaterial," Appl. Phys. Lett. 97, 134106 (2010).

${ }^{7}$ J. S. Bell, I. R. Summers, A. R. J. Murray, E. Hendry, J. R. Sambles and A. P. Hibbins, "Low acoustic transmittance through a holey structure," Phys. Rev. B. 85, 214305 (2012).

${ }^{8}$ L. E. Kinsler, A. R. Frey, A. B. Coppens and J. V. Sanders, Fundamentals of Acoustics, 3rd ed. (John Wiley and Sons, New York, 2000), pp. 200-216. 